\title{
Physicochemical properties of oat beta-glucan influence its LDL cholesterol lowering effect in human subjects
}

\author{
T. M. S. Wolever ${ }^{1}$, S. M. Tosh ${ }^{2}$, A. L. Gibbs ${ }^{3}$, J. Brand-Miller ${ }^{4}$, A. M. Duncan ${ }^{5}$, V. Hart ${ }^{6}$, \\ B. Lamarche ${ }^{7}$, B. Thomson ${ }^{3}$, R. Duss ${ }^{8}$ and P. J. Wood ${ }^{2}$ \\ ${ }^{1}$ Glycemic Index Laboratories Inc., Toronto, ON, Canada, ${ }^{2}$ Agriculture Agri-Foods Canada, Guelph, ON, Canada, \\ ${ }^{3}$ Department of Statistics, University of Toronto, Toronto, ON, Canada, ${ }^{4}$ Molecular and Microbial Biosciences, University \\ of Sydney, Sydney, Australia, ${ }^{5}$ Human Health and Nutritional Sciences, University of Guelph, Guelph, ON, Canada, \\ ${ }^{6}$ Reading Scientific Services Ltd, Reading, UK, ${ }^{7} I N A F$, Laval University, Laval, QC, Canada and ${ }^{8}$ CreaNutrition, \\ $A G$, Zug, Switzerland
}

Daily consumption of $3 \mathrm{~g}$ oat $\beta$-glucan is considered sufficient to lower serum LDL cholesterol (LDL-C), but not all studies show an effect. The ability of oat $\beta$-glucan to reduce LDL-C is thought to depend on viscosity which is controlled by the molecular weight (MW) and the amount of oat $\beta$-glucan solubilized in the intestinal lumen $(\mathrm{C})$, but this has not been demonstrated in human subjects.

Therefore, our two primary objectives were to determine if consuming $3 \mathrm{~g}$ high-MW oat- $\beta$-glucan daily reduced LDL-C, and if LDL-Clowering was related to $\log (\mathrm{MW} \times \mathrm{C})$ of oat- $\beta$-glucan. To address these objectives, we conducted a randomized, controlled, double-blind parallel design clinical trial in two contract-research-organisations and three university nutrition research centres in Canada, Australia and UK. A volunteer sample of healthy subjects with LDL-C $\geq 3.0$ and $\leq 5.0 \mathrm{mmol} / \mathrm{l}$ ( $n 786$ screened, $n 400$ ineligible, $n 19$ refused, $n 367$ randomized, $n 345$ completed) were randomly assigned by the computer to receive one of five treatments. Subjects consumed cereal containing wheat fibre $(n$ 87) or a total of $3 \mathrm{~g}$ high-MW $(n$ 86), $4 \mathrm{~g}$ medium-MW $(n$ 67), $3 \mathrm{~g}$ medium-MW $(n$ 64) or $4 \mathrm{~g}$ low-MW $(n$ 63) oat $\beta$-glucan daily (OatWell ${ }^{\circledR}$, divided doses, twice-daily) for 4 weeks. Using an intent-to-treat analysis, serum-LDL-C concentration after 4 weeks was compared between treatments after adjusting for baseline LDL-C.

After 4 weeks, LDL-C on $3 \mathrm{~g}$ high-MW oat $\beta$-glucan cereal was less than on wheat-fibre cereal by $0.21 \mathrm{mmol} / 1(95 \% \mathrm{CI}$; -0.11 , $-0.30, P=0.0023)$. By analysis of covariance $\log (\mathrm{MW} \times \mathrm{C})$ was a significant determinant of week 4 LDL-C-cholesterol $(P=0.003)$. The treatment effect was not significantly influenced by age, sex, study centre or baseline LDL-C.

It was concluded that consuming only $3 \mathrm{~g}$ high-MW oat $\beta$-glucan daily in a ready-to-eat cereal reduced LDL-C by $0.2 \mathrm{mmol} / \mathrm{l}$; efficacy was reduced in cereals containing oat $\beta$-glucan with low MW. Thus, the physicochemical properties of oat $\beta$-glucan should be considered when assessing the cholesterol-lowering ability of oat-containing products.

The trial was registered at www.clinicaltrials.gov NCT00981981.

Funding was provide by the Swedish Governmental Agency for Innovations Systems and CreaNutrition. 